\title{
The Impact of Antipsychotic Dose Reduction on Clinical Outcomes and Health Care Resource Use Among Medicare Patients with Schizophrenia
}

\author{
Miriam L. Zichlin ${ }^{1} \cdot$ Fan $^{M^{1}}{ }^{1} \cdot$ Sam Leo $^{2} \cdot$ Rajeev Ayyagari ${ }^{1}$
}

Accepted: 6 July 2021 / Published online: 4 September 2021

(c) The Author(s) 2021

\begin{abstract}
Background Antipsychotic medications are used to treat schizophrenia and may be associated with adverse effects, including tardive dyskinesia (TD), following prolonged use or upon changes in dosing regimen.

Objective This retrospective analysis evaluated the burden of antipsychotic dose reduction in Medicare patients with schizophrenia.

Methods This matched cohort study used Medicare claims data (2006-2017) analyzed for patients with schizophrenia and two or more claims for antipsychotics, with one or more antipsychotic monotherapy period $\geq 90$ days. Cohorts were defined for patients with antipsychotic dose reductions $\geq 10 \%$ and stable doses. A separate analysis was conducted using patients with dose reductions $\geq 30 \%$. Outcomes included all-cause emergency room (ER) visits, all-cause inpatient visits, schizophrenia relapse, other psychiatric relapse, and TD diagnosis. Covariates included age, disease duration, comorbidities, and medication use.

Results The analysis included 276,030 patients with $\geq 10 \%$ dose reductions and 211,575 patients with $\geq 30 \%$ dose reductions. Patient characteristics were balanced between cohorts. Patients with $\geq 10 \%$ or $\geq 30 \%$ dose reductions had a shorter time to ER visit, inpatient visit, schizophrenia relapse, other psychiatric relapse, and TD diagnosis versus those receiving stable doses (all $p<0.001$ ). Significance was maintained when unmatched baseline characteristics were adjusted.

Conclusions Patients with antipsychotic dose reductions may be at risk for increased ER visits, increased hospitalizations, and significant unfavorable mental health-related clinical outcomes, suggesting that dose reduction may increase overall health care burden in some patients with schizophrenia. This work highlights the need for alternative strategies in the management of patients with TD.
\end{abstract}

\section{Key Points}

Medicare patients with antipsychotic dose reductions may have increased health care resource use and be at increased risk for mental health-related clinical outcomes.

Antipsychotic dose reductions may increase overall health care burden for some patients with schizophrenia.

Rajeev Ayyagari

rajeev.ayyagari@analysisgroup.com

1 Analysis Group, Inc., 111 Huntington Avenue, Fourteenth Floor, Boston, MA 02199, USA

2 Teva Pharmaceuticals, Parsippany, NJ, USA

\section{Introduction}

Schizophrenia is a psychiatric disorder commonly associated with social and occupational impairments [1]. People with schizophrenia experience positive symptoms (e.g., hallucinations, delusions, disorganized speech), negative symptoms (e.g., flattened affect, avolition, alogia), cognitive impairment, and mood disorders. These symptoms are often debilitating and contribute to the high economic burden associated with schizophrenia [2].

Antipsychotic medications are the mainstay of treatment for schizophrenia [3]. Despite their efficacy in treating schizophrenic symptoms, antipsychotics are associated with significant adverse effects such as extrapyramidal symptoms (e.g., dystonia, akathisia, parkinsonism) and tardive dyskinesia (TD) [4]. TD, in particular, is a potentially serious hyperkinetic movement disorder that occurs 
following prolonged use of dopamine-receptor antagonists [5-7] and can also emerge as a result of antipsychotic dosage changes or treatment discontinuation [8]. TD occurs in approximately $20-50 \%$ of patients treated with antipsychotics and is characterized by involuntary movements of the mouth, trunk, and limbs that are typically stereotypic, choreiform, or dystonic and can be socially stigmatizing, with negative effects on quality of life [7]. Although the incidence of TD has declined in recent years with the widespread use of atypical antipsychotics [6], the risk of developing TD remains clinically significant [9].

Although two vesicular monoamine transporter 2 (VMAT2) inhibitors have been approved by the US FDA for the treatment of TD, health care providers may attempt antipsychotic dose reductions as a means to treat TD despite limited data supporting the efficacy and safety of this approach [10]. Dose reductions may lead to an increased risk of certain forms of TD, including withdrawal-emergent dyskinesia [8], as well as psychiatric relapse [11], which can increase the likelihood of hospitalization, treatment resistance, cognitive impairment, incarceration, personal distress, and thwarted rehabilitation efforts [12].

Evidence on the efficacy of antipsychotic dose reduction or withdrawal for managing TD remains limited $[10,13]$. Recent retrospective cohort studies have found that antipsychotic dose reductions in Medicaid patients aged $\geq 18$ years with schizophrenia, bipolar disorder, or major depressive disorder led to significant increases in both all-cause and mental health-related hospitalizations $[14,15]$. Since there is a dearth of evidence discussing antipsychotic dose reduction for the management of TD in the elderly population, this study aimed to evaluate the impact of antipsychotic dose reductions on all-cause health care resource use (HRU) and mental health-related clinical outcomes in Medicare patients with schizophrenia.

\section{Methods}

\subsection{Data Source}

This retrospective matched cohort analysis used de-identified patient data from the $100 \%$ Medicare database + Part D linkage from 1 January 2006 to 31 December 2017. The data comprised enrollment and claims history from Medicare Part A (institutional services, including inpatient and outpatient claims), Medicare Part B (non-institutional services, including carrier and medical equipment services), and Medicare Part D (prescription drug claims). Patients can be eligible for Medicare due to age ( $\geq 65$ years), disability, or end-stage renal disease. This study received an exemption from the New England Institutional Review Board.

\subsection{Patients}

Patients were eligible for inclusion in the study if they had one or more diagnoses of schizophrenia (International Classification of Diseases, Ninth Revision, Clinical Modification [ICD-9-CM] code 295.xx or International Classification of Diseases, Tenth Revision, Clinical Modification [ICD-10-CM] codes F20.x or F25.x). Following their first observed diagnosis of schizophrenia, patients were required to have two or more pharmacy claims for oral antipsychotics, one or more antipsychotic monotherapy treatment period lasting $\geq 90$ days, and $\geq 6$ months of continuous Medicare eligibility prior to the index date (baseline period), which was defined as the first antipsychotic prescription date following this $\geq 90$-day period. If a patient had multiple $\geq 90$-day periods of antipsychotic monotherapy, the final index date was randomly selected from all eligible index dates. Patients were excluded from the study if they received treatment with long-acting injectable antipsychotics or concurrent antipsychotics during the observation period, which ended at the earliest of the end of continuous eligibility, dose escalation, medication change, or 2 years after the index date.

Patients were classified into two cohorts based on patterns of antipsychotic medication use: patients who experienced $\mathrm{a} \geq 10 \%$ dose reduction in antipsychotic medication during the $\geq 90$-day period of antipsychotic monotherapy, and patients who continued receiving a stable antipsychotic dose, defined as a dose reduction $<10 \%$. Cohorts were matched 1:1, without replacement, based on age, sex, typical versus atypical antipsychotic use, and index year. Sensitivity analyses were conducted to compare matched cohorts with $\geq 30 \%$ dose reductions or those who continued on a stable dose, defined as dose reductions $<30 \%$.

\subsection{Outcomes}

Patients were assessed for all-cause HRU outcomes, including emergency room (ER) and inpatient visits, as well as mental health-related clinical outcomes, including schizophrenia relapse, other psychiatric relapse, and TD diagnosis, during the observation period (i.e., end of continuous eligibility, dose escalation, medication change, or 2 years after the index date). Schizophrenia relapse was defined as an ER or inpatient visit for schizophrenia, and other psychiatric relapse was defined as an ER or inpatient visit for psychiatric conditions excluding schizophrenia (i.e., substance-related and addictive, depressive, bipolar, trauma- and stressorrelated, anxiety, sleep-wake, personality, and other psychotic disorders). TD diagnosis corresponded to ICD-9-CM code 333.85 (subacute dyskinesia due to drugs) or ICD-10-CM code G24.01 (drug-induced subacute dyskinesia). 


\subsection{Statistical Analyses}

Differences between cohorts were evaluated using Wilcoxon signed-rank tests for continuous variables and McNemar's tests for dichotomous variables. Kaplan-Meier analyses were used to estimate median times to HRU and mental health-related clinical outcomes. Cohorts were compared using log-rank tests. Patients with TD at baseline were excluded from the TD analysis. Multivariable Cox proportional hazard models were used to compare outcomes between cohorts after adjusting for unmatched baseline characteristics, such as age, disease duration, Charlson Comorbidity Index (CCI) score, psychiatric comorbidity profile, use of psychotherapy, and use of psychiatric medications. In separate analyses, results were analyzed in subgroups stratified by patient age ( $\geq 65$ years vs. $<65$ years) at the index date to compare outcomes according to Medicare eligibility (i.e., age vs. disability, respectively).

\section{Results}

\subsection{Patients}

Overall, 1,406,070 patients were diagnosed with schizophrenia during the study period. In total, 276,030 patients who met the final inclusion criteria were included in the $\geq 10 \%$ antipsychotic dose reduction cohort and matched 1:1 with patients receiving stable antipsychotic doses. Of these patients, 211,575 had a $\geq 30 \%$ dose reduction (Fig. 1 in Online Resource 1). Distributions of age, sex, index drug (typical vs. atypical antipsychotic), and index year were comparable between patients in the $\geq 10 \%$ antipsychotic dose reduction cohort and patients receiving stable doses (Table 1). Across cohorts, the mean age was 56.6 years, the proportion of male patients was $49.8 \%$, and the proportion of patients using atypical antipsychotics was $87.7 \%$. Patients with $\mathrm{a} \geq 10 \%$ antipsychotic dose reduction had a longer mean duration of schizophrenia (32.7 months vs. 23.5 months) and a shorter mean duration of follow-up time (5.0 months vs. 9.1 months) compared with patients receiving stable doses. Differences in comorbidity profiles indicated that patients with $\geq 10 \%$ dose reductions had lower rates of psychiatric comorbidities and substance use disorders than those receiving stable doses, with the exception of TD. At baseline, patients receiving stable doses experienced slightly more chronic pulmonary disease, while patients with $\geq 10 \%$ dose reductions experienced slightly more peripheral vascular disease. Baseline characteristics for the matched cohorts with $\geq 30 \%$ dose reductions or stable doses (dose reductions $<30 \%$ ) were generally similar to those seen in the matched cohorts with $\geq 10 \%$ dose reductions or stable doses (dose reductions $<10 \%$ ). The 10 most frequently used antipsychotics were the same between the $\geq 10 \%$ and $\geq 30 \%$ dose reduction and stable dose cohorts (Table 1 in Online Resource 1). In addition, baseline characteristics in both analyses, stratified by age $\geq 65$ years and $<65$ years, were generally similar to those of the overall population, with some exceptions (Tables 2 and 3 in Online Resource 1). Dose distributions for the most frequently used antipsychotics stratified by age are shown in Tables 4 and 5 in Online Resource 1.

\subsection{Outcomes}

Patients in the $\geq 10 \%$ dose reduction cohort had a shorter time to all-cause ER visits, all-cause inpatient visits, schizophrenia relapse, and other psychiatric relapse (excluding schizophrenia) compared with those receiving stable doses (Figs. 1, 2, 3, 4, respectively). Median time to the all-cause ER visit was 11.2 months for both the $\geq 10 \%$ dose reduction and stable dose cohorts. Median time to the allcause inpatient visit, schizophrenia relapse, other psychiatric relapse, and TD diagnosis was not reached for both cohorts. After adjusting for differences in unmatched baseline characteristics, patients with $a \geq 10 \%$ dose reduction showed a significantly increased risk for the all-cause ER visit [hazard ratio (HR) 1.08, 95\% confidence interval (CI) 1.07-1.09; $p<0.001$ ], all-cause inpatient visit (HR 1.22, 95\% CI $1.21-1.23 ; p<0.001$ ), schizophrenia relapse (HR 1.24, 95\% CI 1.22-1.26; $p<0.001)$, other psychiatric relapse (HR $1.13,95 \%$ CI $1.12-1.15 ; p<0.001$ ), and TD diagnosis (HR 1.43 , 95\% CI $1.32-1.54 ; p<0.001$ ) (Table 2). This was associated with an $8-22 \%$ increase in the risk of experiencing an all-cause HRU outcome (ER or inpatient visit) and a $13-43 \%$ increase in the risk of experiencing a mental health-related clinical outcome (schizophrenia relapse, other psychiatric relapse, or TD diagnosis).

Patients in the $\geq 30 \%$ dose reduction cohort also had a shorter time to all-cause ER visits, all-cause inpatient visits, schizophrenia relapse, and other psychiatric relapse compared with those receiving stable doses (Figs. 1, 2, 3, 4, respectively). Median time to the all-cause ER visit was 10.2 and 11.1 months for the $\geq 30 \%$ dose reduction and stable dose cohorts, respectively. Median time to the all-cause inpatient visit, schizophrenia relapse, and TD diagnosis was not reached. Median time to other psychiatric relapse was 23.0 months for the $\geq 30 \%$ dose reduction cohort and was not reached for the stable dose cohort. After adjusting for differences in unmatched baseline characteristics, patients with $a \geq 30 \%$ dose reduction showed significantly increased risk for the all-cause ER visit (HR 1.11, 95\% CI $1.10-1.12 ; p<0.001$ ), all-cause inpatient visit (HR 1.26, 95\% CI 1.24-1.27; $p<0.001$ ), schizophrenia relapse (HR 1.25, 95\% CI 1.23-1.27; $p<0.001$ ), other psychiatric relapse (HR 1.17, 95\% CI 1.16-1.19; $p<0.001$ ), and TD 
Table 1 Baseline characteristics in the $\geq 10 \%$ and $\geq 30 \%$ antipsychotic dose reduction cohorts in the overall population

\begin{tabular}{|c|c|c|c|c|}
\hline \multirow[t]{2}{*}{ Characteristic } & \multicolumn{2}{|c|}{$\geq 10 \%$ dose reduction cohorts } & \multicolumn{2}{|c|}{$\geq 30 \%$ dose reduction cohorts } \\
\hline & $\begin{array}{l}\text { Dose reduction } \\
{[n=276,030]}\end{array}$ & $\begin{array}{l}\text { Stable dose } \\
{[n=276,030]}\end{array}$ & $\begin{array}{l}\text { Dose reduction } \\
{[n=211,575]}\end{array}$ & $\begin{array}{l}\text { Stable dose } \\
{[n=211,575]}\end{array}$ \\
\hline Age, years $[\text { mean }(\mathrm{SD})]^{\mathrm{a}}$ & $56.6(15.6)$ & $56.6(15.6)$ & $57.0(15.7)$ & $56.9(15.7)$ \\
\hline Schizophrenia duration, months [mean (SD) $]^{\mathrm{a}}$ & $32.7(21.3)$ & $23.5(21.5)$ & $32.4(21.3)$ & $23.5(21.6)$ \\
\hline Sex, male & $137,334(49.8)$ & $137,334(49.8)$ & $103,817(49.1)$ & $103,817(49.1)$ \\
\hline Duration of follow-up, months [mean (SD)] ${ }^{\mathrm{a}}$ & $5.0(6.9)$ & $9.1(8.7)$ & $4.5(6.6)$ & $9.1(8.6)$ \\
\hline \multicolumn{5}{|l|}{ Index drug class } \\
\hline Typical antipsychotic & $33,942(12.3)$ & $33,942(12.3)$ & $26,548(12.5)$ & $26,548(12.5)$ \\
\hline Atypical antipsychotic & $242,088(87.7)$ & $242,088(87.7)$ & $185,027(87.5)$ & $185,027(87.5)$ \\
\hline \multicolumn{5}{|l|}{ Index year } \\
\hline 2011 & $18,132(6.6)$ & $18,132(6.6)$ & $13,603(6.4)$ & $13,603(6.4)$ \\
\hline 2012 & $42,148(15.3)$ & $42,148(15.3)$ & $32,129(15.2)$ & $32,129(15.2)$ \\
\hline 2013 & $42,737(15.5)$ & $42,737(15.5)$ & $32,858(15.5)$ & $32,858(15.5)$ \\
\hline 2014 & $42,806(15.5)$ & $42,806(15.5)$ & $32,926(15.6)$ & $32,926(15.6)$ \\
\hline 2015 & $42,145(15.3)$ & $42,145(15.3)$ & $32,282(15.3)$ & $32,282(15.3)$ \\
\hline 2016 & $43,210(15.7)$ & $43,210(15.7)$ & $33,174(15.7)$ & $33,174(15.7)$ \\
\hline 2017 & $44,852(16.2)$ & $44,852(16.2)$ & $34,603(16.4)$ & $34,603(16.4)$ \\
\hline \multicolumn{5}{|l|}{ Comorbidity profile } \\
\hline Substance-related and addictive disorders ${ }^{\mathrm{a}}$ & $72,508(26.3)$ & $84,623(30.7)$ & $56,789(26.8)$ & $64,341(30.4)$ \\
\hline Anxiety disorders ${ }^{\mathrm{a}}$ & $75,593(27.4)$ & $86,252(31.2)$ & $60,034(28.4)$ & $66,460(31.4)$ \\
\hline Bipolar disorders $^{\mathrm{a}}$ & $84,331(30.6)$ & $90,413(32.8)$ & $66,073(31.2)$ & $68,996(32.6)$ \\
\hline Depressive disorders $^{\mathrm{a}}$ & $98,003(35.5)$ & $111,274(40.3)$ & $77,950(36.8)$ & $85,710(40.5)$ \\
\hline Personality disorders ${ }^{\mathrm{a}}$ & $13,426(4.9)$ & $14,441(5.2)$ & $10,410(4.9)$ & $11,046(5.2)$ \\
\hline $\begin{array}{l}\text { Schizophrenia-spectrum disorders } \\
\text { (excluding schizophrenia) }^{\mathrm{a}}\end{array}$ & $50,792(18.4)$ & $60,633(22.0)$ & $40,311(19.1)$ & $46,762(22.1)$ \\
\hline Sleep-wake disorders ${ }^{\mathrm{a}}$ & $43,368(15.7)$ & $48,852(17.7)$ & $34,212(16.2)$ & $37,552(17.7)$ \\
\hline $\mathrm{TD}^{\mathrm{a}}$ & $2778(1.0)$ & $2488(0.9)$ & $2094(1.0)$ & $1929(0.9)$ \\
\hline Trauma- and stressor-related disorders ${ }^{\mathrm{a}}$ & $20,009(7.2)$ & $23,280(8.4)$ & $15,930(7.5)$ & $17,862(8.4)$ \\
\hline CCI score [mean (SD)] & $1.3(1.8)$ & $1.4(1.9)$ & $1.4(1.9)$ & $1.4(1.9)$ \\
\hline AIDS/HIV & $2812(1.0)$ & $3638(1.3)$ & $2255(1.1)$ & $2760(1.3)$ \\
\hline Cancer $^{\mathrm{a}}$ & $11,017(4.0)$ & $12,206(4.4)$ & $8601(4.1)$ & $9552(4.5)$ \\
\hline Cerebrovascular disease $^{\mathrm{a}}$ & $28,628(10.4)$ & $30,384(11.0)$ & $23,156(10.9)$ & $23,711(11.2)$ \\
\hline Congestive heart failure $^{\mathrm{a}}$ & $29,494(10.7)$ & $32,278(11.7)$ & $23,924(11.3)$ & $25,160(11.9)$ \\
\hline Chronic pulmonary disease $^{\mathrm{a}}$ & $76,219(27.6)$ & $80,209(29.1)$ & $59,860(28.3)$ & $61,771(29.2)$ \\
\hline Dementia $^{\mathrm{a}}$ & $39,795(14.4)$ & $37,633(13.6)$ & $32,150(15.2)$ & $29,926(14.1)$ \\
\hline Diabetes with chronic complications ${ }^{\mathrm{a}}$ & $30,132(10.9)$ & $29,615(10.7)$ & $23,690(11.2)$ & $22,794(10.8)$ \\
\hline Diabetes without chronic complications ${ }^{\mathrm{a}}$ & $63,767(23.1)$ & $61,593(22.3)$ & $49,081(23.2)$ & $47,381(22.4)$ \\
\hline Hemiplegia or paraplegia & $6626(2.4)$ & $6705(2.4)$ & $5286(2.5)$ & $5126(2.4)$ \\
\hline Mild liver disease $^{\mathrm{a}}$ & $14,329(5.2)$ & $15,950(5.8)$ & $11,309(5.3)$ & $12,093(5.7)$ \\
\hline Metastatic solid tumor ${ }^{\mathrm{a}}$ & $1851(0.7)$ & $2723(1.0)$ & $1480(0.7)$ & $2081(1.0)$ \\
\hline Myocardial infarction $^{\mathrm{a}}$ & $7371(2.7)$ & $9810(3.6)$ & $6030(2.9)$ & $7584(3.6)$ \\
\hline Moderate or severe liver disease ${ }^{\mathrm{a}}$ & $1808(0.7)$ & $2081(0.8)$ & $1490(0.7)$ & $1611(0.8)$ \\
\hline Peptic ulcer disease ${ }^{\mathrm{a}}$ & $3706(1.3)$ & $4327(1.6)$ & $2984(1.4)$ & $3321(1.6)$ \\
\hline Peripheral vascular disease ${ }^{\mathrm{a}}$ & $46,715(16.9)$ & $41,277(15.0)$ & $36,961(17.5)$ & $32,174(15.2)$ \\
\hline Renal disease $^{\mathrm{a}}$ & $26,030(9.4)$ & $27,345(9.9)$ & $20,718(9.8)$ & $21,285(10.1)$ \\
\hline Rheumatic disease $^{\mathrm{a}}$ & $5786(2.1)$ & $7012(2.5)$ & $4611(2.2)$ & $5427(2.6)$ \\
\hline \multicolumn{5}{|l|}{ Additional psychiatric medications } \\
\hline ADHD medication ${ }^{\mathrm{a}}$ & $7476(2.7)$ & $8349(3.0)$ & $5836(2.8)$ & $6304(3.0)$ \\
\hline Anticholinergic $^{\mathrm{a}}$ & $62,729(22.7)$ & $54,139(19.6)$ & $47,200(22.3)$ & $41,198(19.5)$ \\
\hline
\end{tabular}


Table 1 (continued)

\begin{tabular}{|c|c|c|c|c|}
\hline \multirow[t]{2}{*}{ Characteristic } & \multicolumn{2}{|c|}{$\geq 10 \%$ dose reduction cohorts } & \multicolumn{2}{|c|}{$\geq 30 \%$ dose reduction cohorts } \\
\hline & $\begin{array}{l}\text { Dose reduction } \\
{[n=276,030]}\end{array}$ & $\begin{array}{l}\text { Stable dose } \\
{[n=276,030]}\end{array}$ & $\begin{array}{l}\text { Dose reduction } \\
{[n=211,575]}\end{array}$ & $\begin{array}{l}\text { Stable dose } \\
{[n=211,575]}\end{array}$ \\
\hline Antidepressant ${ }^{\mathrm{a}}$ & $171,161(62.0)$ & $175,336(63.5)$ & $132,777(62.8)$ & $134,610(63.6)$ \\
\hline Anxiety medication $^{\mathrm{a}}$ & $73,571(26.7)$ & $80,173(29.0)$ & $57,518(27.2)$ & 61,747 (29.2) \\
\hline Mood stabilizer ${ }^{\mathrm{a}}$ & $121,424(44.0)$ & $113,000(40.9)$ & $93,582(44.2)$ & $86,429(40.9)$ \\
\hline Sedative $^{\mathrm{a}}$ & $27,856(10.1)$ & $33,144(12.0)$ & $22,219(10.5)$ & $25,471(12.0)$ \\
\hline
\end{tabular}

Data are expressed as $n(\%)$ unless otherwise specified

$A D H D$ attention-deficit hyperactivity disorder, AIDS acquired immunodeficiency syndrome, $C C I$ Charlson Comorbidity Index, $H I V$ human immunodeficiency virus, $S D$ standard deviation, $T D$ tardive dyskinesia

${ }^{\mathrm{a}} p<0.05$ for dose reductions versus stable doses in both dose reduction cohorts

diagnosis (HR 1.39, 95\% CI 1.26-1.52; $p<0.001$ ) (Table 2). This was associated with an 11-26\% increase in the risk of experiencing an all-cause HRU outcome and a 17-39\% increase in the risk of experiencing a mental health-related clinical outcome.

Age-stratified results for patients with $\geq 10 \%$ and $\geq 30 \%$ dose reductions were generally consistent with the overall results, with generally larger HRs among patients aged $<65$ years compared with patients aged $\geq 65$ years (Tables 6 and 7 in Online Resource 1). Patients aged $<65$ years with dose reductions experienced shorter time to the all-cause ER visit, all-cause inpatient visit, schizophrenia relapse, and other psychiatric relapse compared with patients receiving stable doses. Although patients aged $\geq 65$ years with dose reductions also experienced a shorter time to schizophrenia relapse, the time to the all-cause ER visit, all-cause inpatient visit, and other psychiatric relapse was slightly longer compared with those receiving stable doses (Figs. 2-5 in Online Resource 1, respectively). These findings were seen across both definitions of dose reduction.

\section{Discussion}

Schizophrenia is a chronic and debilitating mental illness that strains health care resources. In particular, inpatient visits account for the largest portion of health care burden, representing approximately $60 \%$ of total direct costs [16]. Furthermore, patients with schizophrenia who experience a relapse have two to five times higher annual mental health costs than those who do not experience a relapse [17]. Although antipsychotic dose reduction is recommended for patients with schizophrenia who are experiencing adverse effects (e.g., sedation, neuroleptic malignant syndrome), even modest dose reductions can contribute to greater HRU for patients and can lead to negative clinical outcomes. In this observational study using Medicare claims data, patients with $\geq 10 \%$ and $\geq 30 \%$ antipsychotic dose reductions demonstrated small but statistically significant increases in both all-cause HRU outcomes (ER and inpatient visits) and mental health-related clinical outcomes (schizophrenia relapse, other psychiatric relapse, and TD diagnosis). The increased risk of TD diagnosis seen in both the $\geq 10 \%$ and $\geq 30 \%$ dose reduction cohorts may be attributed, in part, to the longer mean duration of schizophrenia observed in the dose reduction cohorts versus stable dose cohorts, which may result in a higher cumulative antipsychotic dose, and to withdrawal dyskinesias following dose reduction.

Findings from the present analysis evaluating Medicare beneficiaries are consistent with those reported in a similar study using Medicaid data [15]. The retrospective cohort study of the Medicaid population revealed patients with schizophrenia who underwent $\geq 10 \%$ and $\geq 30 \%$ antipsychotic dose reductions experienced significant increases in both all-cause and mental health-related ER visits and inpatient admissions compared with patients who were treated with stable antipsychotic doses [15]. Taken together, results from both studies suggest that antipsychotic dose reductions may destabilize psychiatric status and lead to increased hospitalization rates in patients with schizophrenia.

As patients can be eligible for Medicare due to age ( $\geq 65$ years), disability, or end-stage renal disease, this study also separately evaluated outcomes for patients aged $\geq 65$ years and $<65$ years to assess whether eligibility reason confounded results. Results among patients aged $<65$ years were largely similar to those of the overall Medicare population, whereas results were mixed among patients aged $\geq 65$ years. Patients aged $<65$ years may be eligible for Medicare due to disability caused by more severe schizophrenia and may therefore be more susceptible to relapse or hospitalization as a result of antipsychotic dose reduction. Patients aged $\geq 65$ years, likely eligible for Medicare due to age, may have a less severe form of the illness.

The strengths of the current study include use of a large claims database to identify Medicare patients with 
A

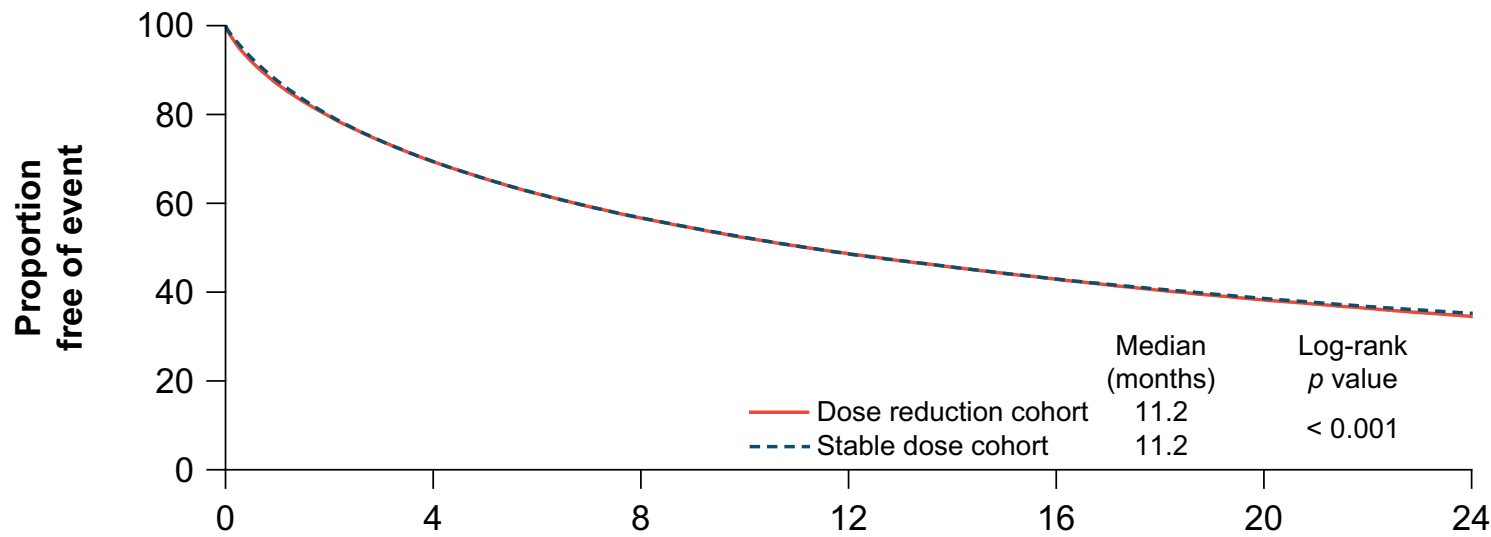

Time (months)

\begin{tabular}{|c|c|c|c|c|c|c|c|}
\hline$n$ at risk & & & & & & & \\
\hline Dose reduction cohort & 276,030 & 68,603 & 38,814 & 25,039 & 17,337 & 12,342 & 8990 \\
\hline Stable dose cohort & 276,030 & 119,895 & 74,855 & 52,401 & 38,963 & 29,806 & 23,373 \\
\hline
\end{tabular}

B

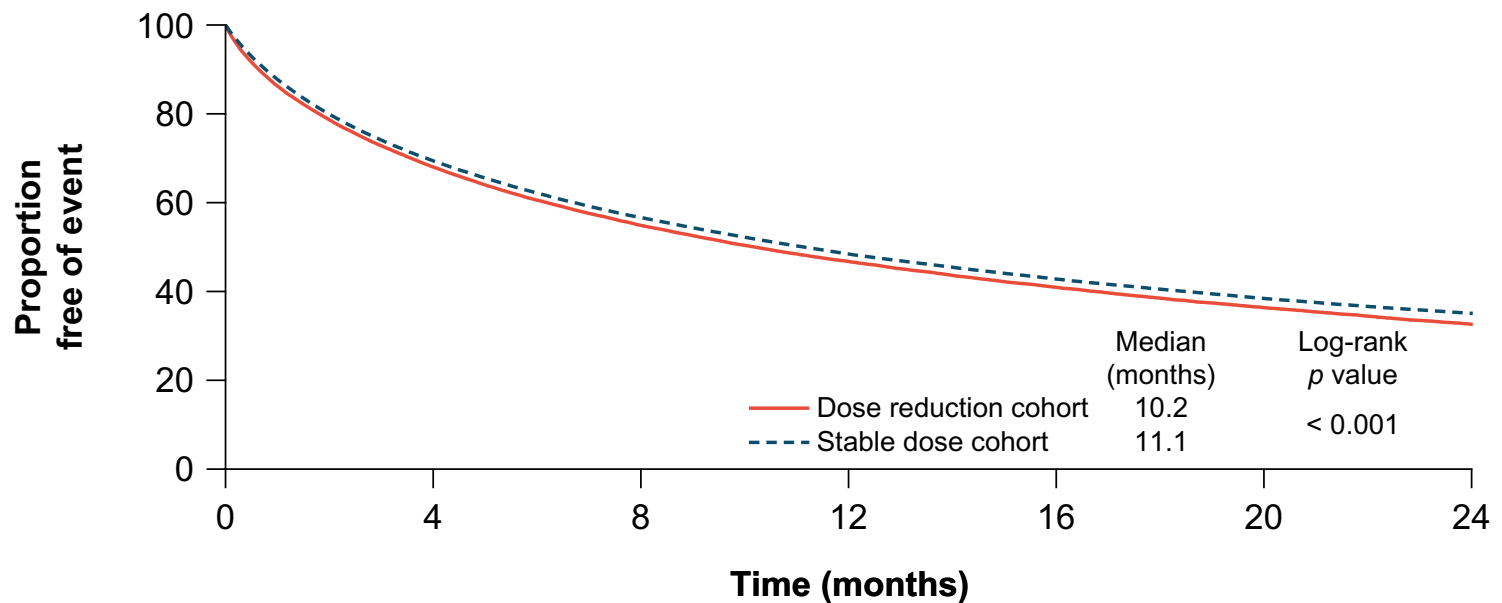

\section{$n$ at risk}

Dose reduction cohort

Stable dose cohort
46,695

91,735
25,635

57,048
16,219
39,816
11,010
29,531
7755

22,591
5582

17,693
Fig. 1 Time to the all-cause emergency room (ER) visit in the $\mathbf{a} \geq 10 \%$ and $\mathbf{b} \geq 30 \%$ dose reduction cohorts in the overall population. These are Kaplan-Meier graphs describing the time to the allcause ER visit in the $\geq 10 \%$ and $\geq 30 \%$ dose reduction and stable dose cohorts in the overall population. The $x$-axis represents time in months at an interval of 4 months, and the $y$-axis represents the pro- portion of patients free of event. The median time to the all-cause ER visit was 11.2 months for both the $\geq 10 \%$ dose reduction and stable dose cohorts, with a $p$ value of $<0.001$. The median time to the allcause ER visit was 10.2 months for the $\geq 30 \%$ dose reduction cohort and 11.1 months for the stable dose cohort, with a $p$ value of $<0.001$

unobserved differences between cohorts. Second, certain comorbidities may have been underestimated due to the use of administrative diagnosis codes. Third, reasons for antipsychotic dose reduction cannot be gleaned from the database, and it is unknown whether doses were reduced due to clinical improvement of schizophrenia or worsening of adverse events. Fourth, the time from first diagnosis of schizophrenia to the index date was used as a proxy for duration of schizophrenia. However, as with 
A

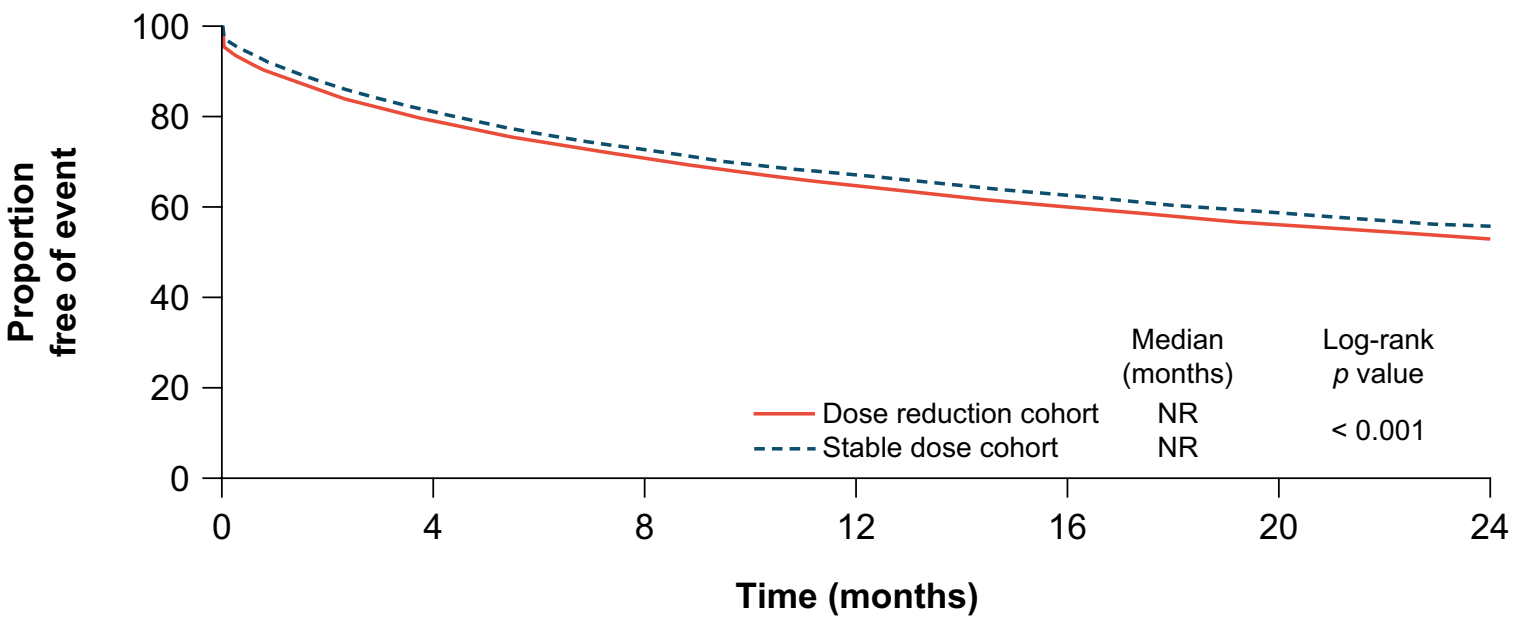

$n$ at risk

Dose reduction cohort

B

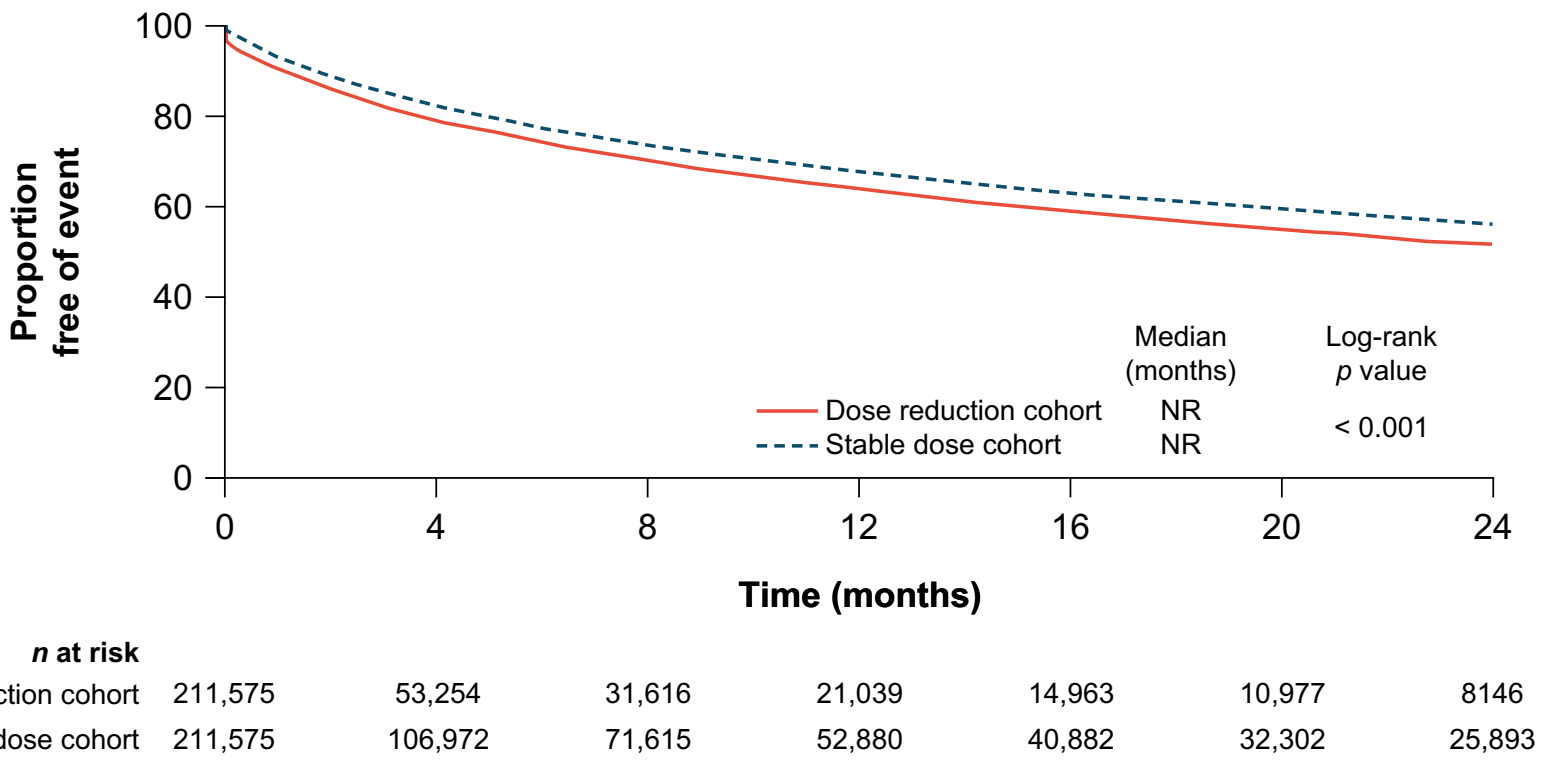

Fig. 2 Time to the all-cause inpatient visit in the $\mathbf{a} \geq 10 \%$ and $\mathbf{b} \geq 30 \%$ dose reduction cohorts in the overall population. These are Kaplan-Meier graphs describing the time to all-cause inpatient visit in the $\geq 10 \%$ and $\geq 30 \%$ dose reduction and stable dose cohorts in the overall population. The $x$-axis represents time in months at an

all retrospective studies of health care claims data, the first observed diagnosis may not have been the patient's first-ever diagnosis for schizophrenia (as the data are left censored). Lastly, results from this analysis may not be generalizable to other patient populations, as the study population was limited to US patients covered by Medicare. interval of 4 months, and the $y$-axis represents the proportion of patients free of event. The median time to the all-cause inpatient visit was not reached (NR) for the $\geq 10 \%$ and $\geq 30 \%$ dose reduction and stable dose cohorts, with $p$ values of $<0.001$ in both comparisons

\section{Conclusion}

This study demonstrated a statistically significant increase in the risk of having an all-cause HRU outcome (ER or inpatient visit) and mental health-related clinical outcome (schizophrenia relapse, other psychiatric relapse, or TD diagnosis) with antipsychotic dose reductions of $\geq 10 \%$ 
A

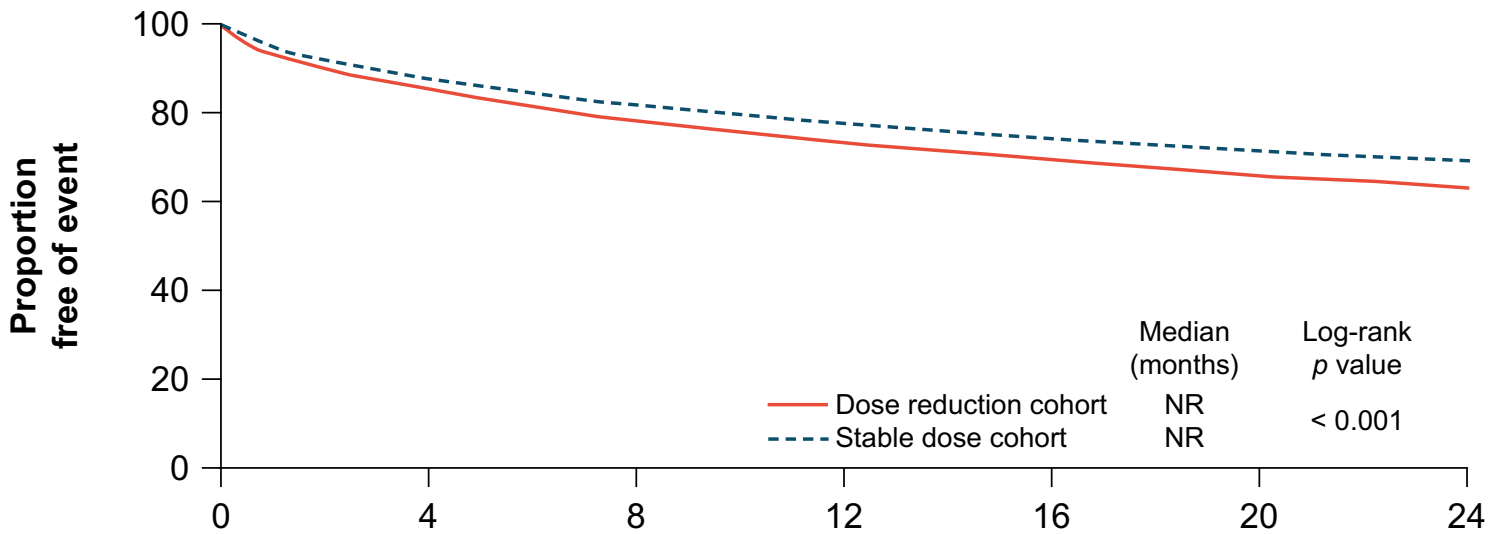

Time (months)

$\begin{array}{rcccccr}\boldsymbol{n} \text { at } \text { risk } & & & & & & \\ \text { Dose reduction cohort } & 276,030 & 81,612 & 50,130 & 34,205 & 24,888 & 18,469 \\ \text { Stable dose cohort } & 276,030 & 146,690 & 100,494 & 75,210 & 58,857 & 46,994\end{array}$

B

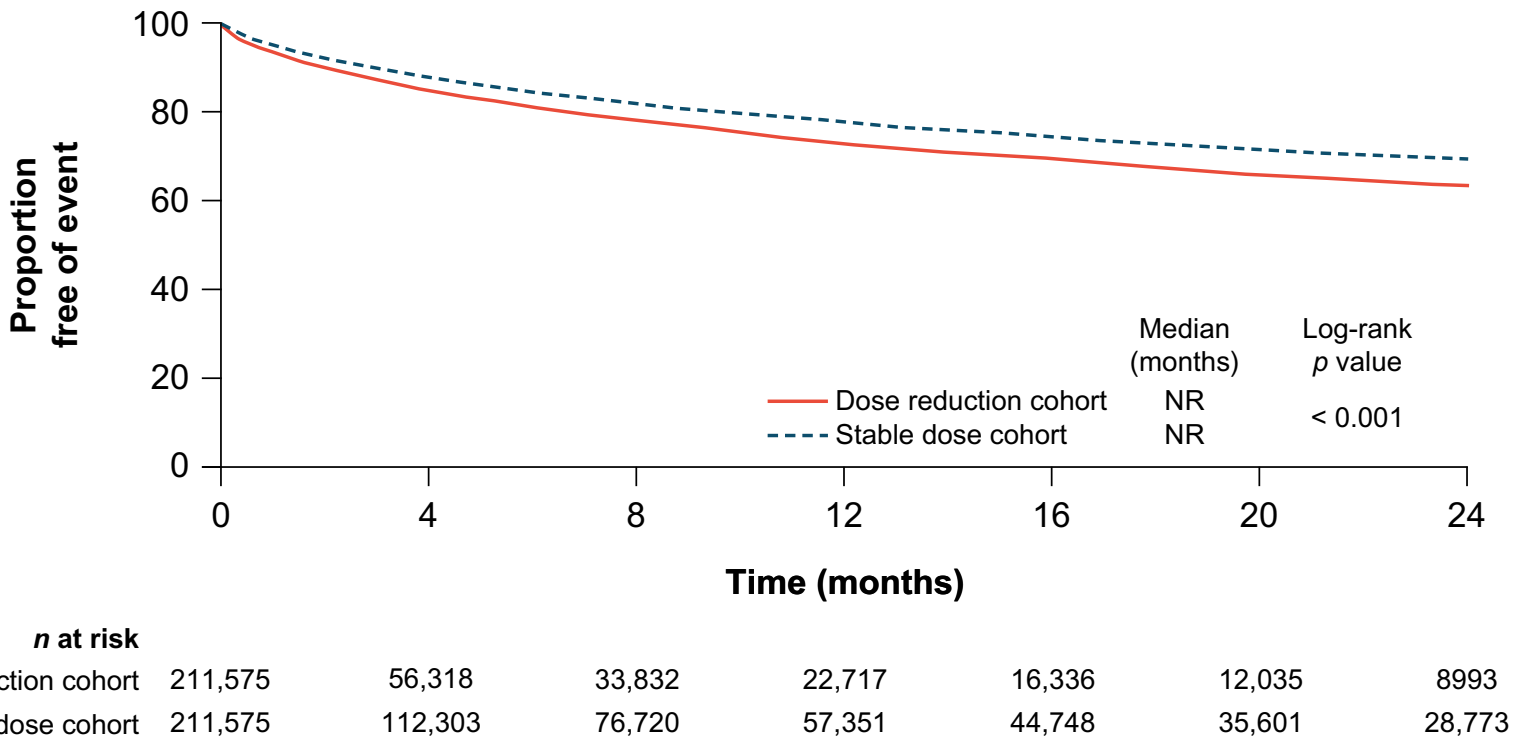

Fig. 3 Time to schizophrenia relapse in the $\mathbf{a} \geq 10 \%$ and $\mathbf{b} \geq 30 \%$ dose reduction cohorts in the overall population. These are Kaplan-Meier graphs describing the time to schizophrenia relapse in the $\geq 10 \%$ and $\geq 30 \%$ dose reduction and stable dose cohorts in the overall population. The $x$-axis represents time in months at an interval

and $\geq 30 \%$ from baseline. $\mathrm{A} \geq 10 \%$ antipsychotic dose reduction was associated with an $8-22 \%$ increase in the risk of having an all-cause HRU outcome and a 13-43\% increase in the risk of having a mental health-related clinical outcome. $\mathrm{A} \geq 30 \%$ antipsychotic dose reduction was associated with an 11-26\% increase in the risk of having an all-cause HRU outcome and a 17-39\% increase in the risk of having a mental health-related clinical outcome. of 4 months, and the $y$-axis represents the proportion of patients free of event. The median time to schizophrenia relapse was not reached (NR) for the $\geq 10 \%$ and $\geq 30 \%$ dose reduction and stable dose cohorts, with $p$ values of $<0.001$ in both comparisons

The clinical meaningfulness of these differences should be considered by health care decision makers, as even seemingly small differences in event rates can have a profound impact on the health and economic burden for both patients and health systems. Nevertheless, decisions for antipsychotic dose reductions should be individualized, with consideration of the potential risks, and remain at the clinician's discretion. Additional research is needed 
A

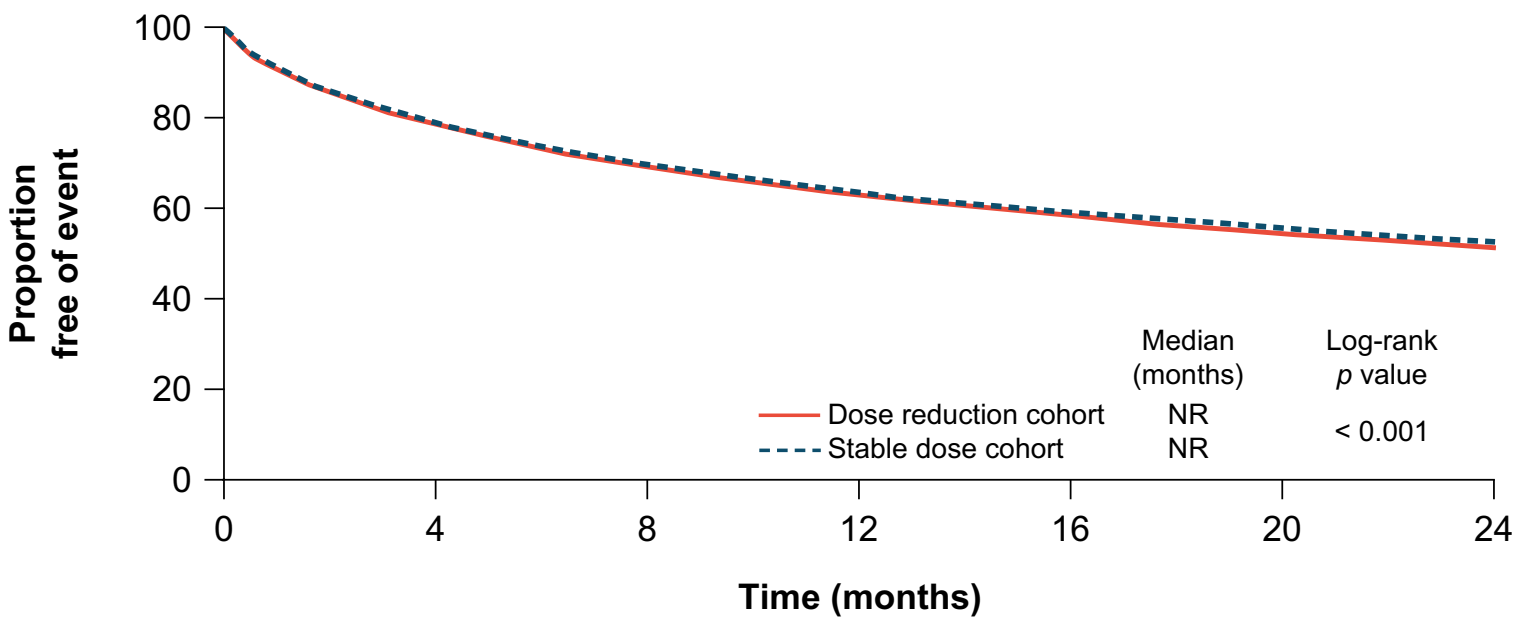

$n$ at risk

Dose reduction cohort

276,030

76,462

45,985

30,973

22,302

16,493

12,381

Stable dose cohort

276,030

134,509

89,201

65,460

50,513

39,874

31,960

B

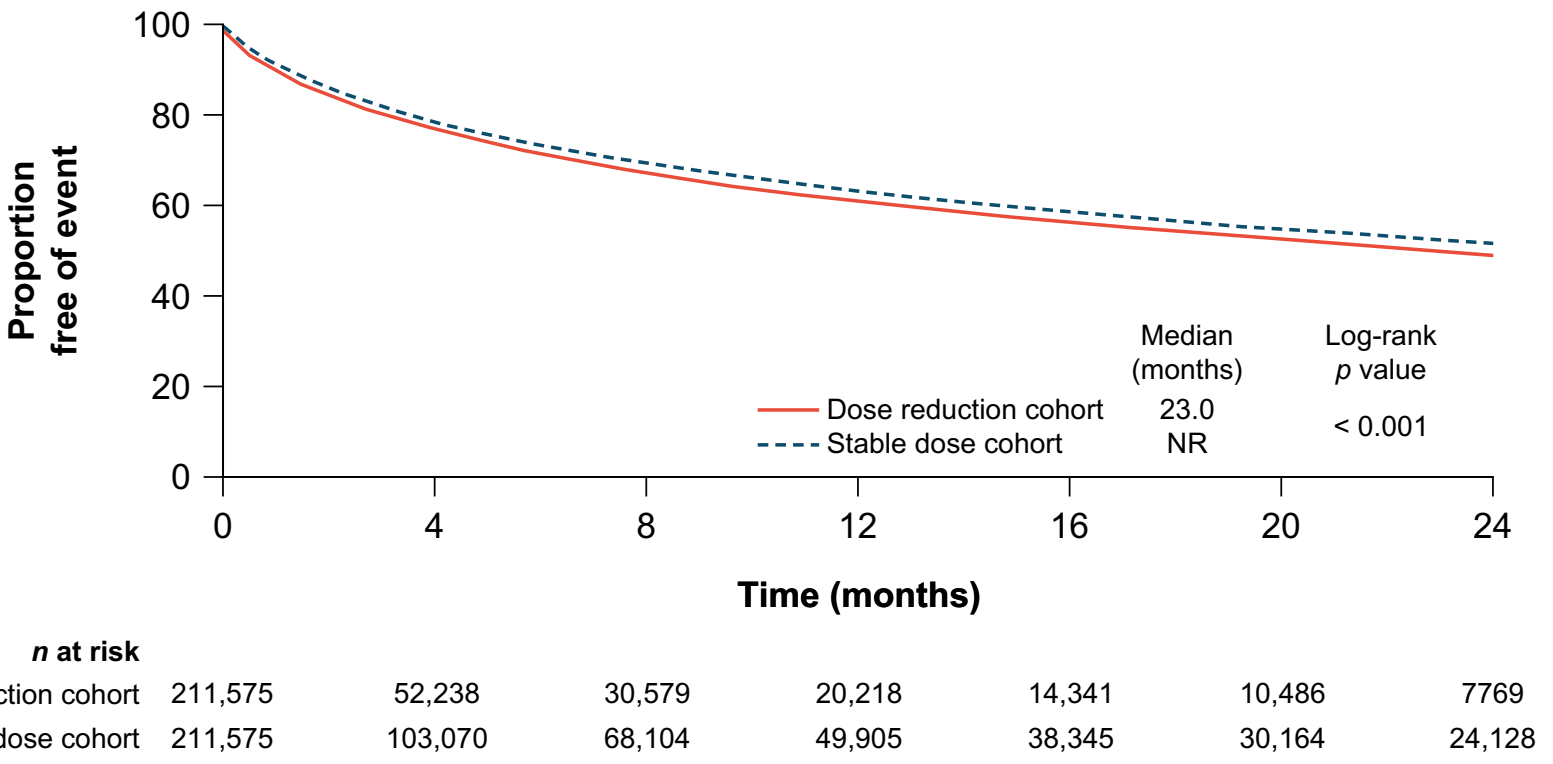

Fig. 4 Time to other psychiatric relapse (excluding schizophrenia) ${ }^{\mathrm{a}}$ in the $\mathbf{a} \geq 10 \%$ and $\mathbf{b} \geq 30 \%$ dose reduction cohorts in the overall population. ${ }^{a}$ Psychiatric relapse was defined as an inpatient admission or ER visit for psychiatric conditions, excluding schizophrenia and schizoaffective disorder. These are Kaplan-Meier graphs describing the time to other psychiatric relapse, excluding schizophrenia, in the $\geq 10 \%$ and $\geq 30 \%$ dose reduction and stable dose cohorts in the

to further understand the value of alternative strategies to antipsychotic dose reduction to address potential adverse overall population. The $x$-axis represents time in months at an interval of 4 months, and the $y$-axis represents the proportion of patients free of event. The median time to other psychiatric relapse was not reached (NR) for both the $\geq 10 \%$ dose reduction and stable dose cohorts, with a $p$ value of $<0.001$. The median time to other psychiatric relapse was 23.0 months for the $\geq 30 \%$ dose reduction cohort and NR for the stable dose cohort, with a $p$ value of $<0.001$

effects that may disrupt necessary maintenance treatment in patients with severe psychiatric disease. 
Table 2 Multivariable Cox regression analyses of the effects of antipsychotic dose reductions in the overall population

\begin{tabular}{|c|c|c|c|c|c|c|}
\hline \multirow[t]{2}{*}{ Outcome } & \multicolumn{3}{|c|}{$\geq 10 \%$ antipsychotic dose reduction } & \multicolumn{3}{|c|}{$\geq 30 \%$ antipsychotic dose reduction } \\
\hline & HR & $95 \% \mathrm{CI}$ & $p$ value & HR & $95 \% \mathrm{CI}$ & $p$ value \\
\hline All-cause ER visit & 1.08 & $1.07-1.09$ & $<0.001$ & 1.11 & $1.10-1.12$ & $<0.001$ \\
\hline All-cause inpatient visit & 1.22 & $1.21-1.23$ & $<0.001$ & 1.26 & $1.24-1.27$ & $<0.001$ \\
\hline Schizophrenia relapse & 1.24 & $1.22-1.26$ & $<0.001$ & 1.25 & $1.23-1.27$ & $<0.001$ \\
\hline $\begin{array}{l}\text { Other psychiatric relapse } \\
\text { (excluding schizophrenia) }\end{array}$ & 1.13 & $1.12-1.15$ & $<0.001$ & 1.17 & $1.16-1.19$ & $<0.001$ \\
\hline TD diagnosis $^{\mathrm{a}}$ & 1.43 & $1.32-1.54$ & $<0.001$ & 1.39 & $1.26-1.52$ & $<0.001$ \\
\hline
\end{tabular}

Covariates included age; disease duration; CCI; substance-related and addictive, anxiety, bipolar and related, depressive, personality, schizophrenia-spectrum (excluding schizophrenia), sleep-wake, trauma-related, and stressor-related disorders; TD; psychotherapy; antidepressants; anticholinergics; sedatives; mood stabilizers; anxiety medications; and ADHD medications

$A D H D$ attention-deficit hyperactivity disorder, $C C I$ Charlson Comorbidity Index, $C I$ confidence interval, $E R$ emergency room, $H R$ hazard ratio, $T D$ tardive dyskinesia

a Patients with TD during the 6-month period prior to the index date were excluded from the TD analyses. For patients with a dose reduction, the index date was defined as the date of the initial dose reduction. For patients receiving a stable dose, the index date was defined as the date of the first prescription fill after the first stable dose monotherapy period of $\geq 90$ days

Supplementary Information The online version contains supplementary material available at https://doi.org/10.1007/s40261-021-01060-3.

Acknowledgements This study was funded by Teva Pharmaceutical Industries Ltd, Petach Tikva, Israel. The authors thank Melanie Chen, PharmD (Cello Health Communications/MedErgy, with funding from Teva Pharmaceutical Industries Inc.), for editorial assistance in the preparation of this report.

\section{Declarations}

Funding This study was funded by Teva Pharmaceutical Industries Ltd, Petach Tikva, Israel.

Conflict of interest Miriam L. Zichlin, Fau Mu, and Rajeev Ayyagari are employees of Analysis Group, Inc. Sam Leo is an employee of Teva Pharmaceuticals.

Ethics approval This study received an exemption from the New England Institutional Review Board.

Consent to participate Not applicable.

Consent for publication Not applicable.

Availability of data and material These data are not publicly available.

Code availability Not applicable.

Author contributions Miriam L. Zichlin, Fan Mu, and Rajeev Ayyagari contributed to the conception and design of the study, analysis and interpretation of the data, and drafting of the manuscript. Sam Leo contributed to the conception and design of the study, interpretation of the data, and drafting of the manuscript. All authors met the International Committee of Medical Journal Editors (ICMJE) criteria for authorship for this article, take responsibility for the integrity of the work as a whole, and have given their approval for this version to be published.

Open Access This article is licensed under a Creative Commons Attribution-NonCommercial 4.0 International License, which permits any non-commercial use, sharing, adaptation, distribution and reproduction in any medium or format, as long as you give appropriate credit to the original author(s) and the source, provide a link to the Creative Commons licence, and indicate if changes were made. The images or other third party material in this article are included in the article's Creative Commons licence, unless indicated otherwise in a credit line to the material. If material is not included in the article's Creative Commons licence and your intended use is not permitted by statutory regulation or exceeds the permitted use, you will need to obtain permission directly from the copyright holder. To view a copy of this licence, visit http://creativecommons.org/licenses/by-nc/4.0/.

\section{References}

1. Tandon R, Gaebel W, Barch DM, Bustillo J, Gur RE, Heckers S, et al. Definition and description of schizophrenia in the DSM-5. Schizophr Res. 2013;150(1):3-10.

2. Cloutier M, Aigbogun MS, Guerin A, Nitulescu R, Ramanakumar AV, Kamat SA, et al. The economic burden of schizophrenia in the United States in 2013. J Clin Psychiatry. 2016;77(6):764-71.

3. Lehman AF, Lieberman JA, Dixon LB, McGlashan TH, Miller AL, Perkins DO, et al. Practice guideline for the treatment of patients with schizophrenia, second edition. Am J Psychiatry. 2004;161 (2 suppl):1-56.

4. Marder SR, Essock SM, Miller AL, Buchanan RW, Casey DE, Davis JM, et al. Physical health monitoring of patients with schizophrenia. Am J Psychiatry. 2004;161(8):1334-49.

5. Aquino CC, Lang AE. Tardive dyskinesia syndromes: current concepts. Parkinsonism Relat Disord. 2014;20(suppl 1):S113-7.

6. Caroff SN, Hurford I, Lybrand J, Campbell EC. Movement disorders induced by antipsychotic drugs: implications of the CATIE schizophrenia trial. Neurol Clin. 2011;29(1):127-48 (viii).

7. Waln O, Jankovic J. An update on tardive dyskinesia: from phenomenology to treatment. Tremor Other Hyperkinet Mov (N Y). 2013;3:1-11.

8. Owens DC. Tardive dyskinesia update: the syndrome. BJPsych Adv. 2018;25(1):57-69.

9. Alexander GC, Gallagher SA, Mascola A, Moloney RM, Stafford RS. Increasing off-label use of antipsychotic medications 
in the United States, 1995-2008. Pharmacoepidemiol Drug Saf. 2011;20(2):177-84.

10. Bergman H, Rathbone J, Agarwal V, Soares-Weiser K. Antipsychotic reduction and/or cessation and antipsychotics as specific treatments for tardive dyskinesia. Cochrane Database Syst Rev. 2018;2:CD000459.

11. Schooler NR. Maintenance medication for schizophrenia: strategies for dose reduction. Schizophr Bull. 1991;17(2):311-24.

12. Sariah AE, Outwater AH, Malima KI. Risk and protective factors for relapse among individuals with schizophrenia: a qualitative study in Dar es Salaam, Tanzania. BMC Psychiatry. 2014;14:240.

13. Bhidayasiri R, Fahn S, Weiner WJ, Gronseth GS, Sullivan KL, Zesiewicz TA, et al. Evidence-based guideline: treatment of tardive syndromes: report of the Guideline Development Subcommittee of the American Academy of Neurology. Neurology. 2013;81(5):463-9.
14. Caroff SN, Mu F, Ayyagari R, Schilling T, Abler V, Carroll B. Hospital utilization rates following antipsychotic dose reductions: implications for tardive dyskinesia. BMC Psychiatry. 2018;18(1):306.

15. Caroff SN, Mu F, Ayyagari R, Schilling T, Abler V, Carroll B. Hospital utilization rates following antipsychotic dose reduction in mood disorders: implications for treatment of tardive dyskinesia. BMC Psychiatry. 2020;20(1):365.

16. Wyatt RJ, Henter I, Leary MC, Taylor E. An economic evaluation of schizophrenia-1991. Soc Psychiatry Psychiatr Epidemiol. 1995;30(5):196-205.

17. Ascher-Svanum H, Zhu B, Faries DE, Salkever D, Slade EP, Peng $X$, et al. The cost of relapse and the predictors of relapse in the treatment of schizophrenia. BMC Psychiatry. 2010;10:2. 